\title{
Fluctuations and the existence of potential in dissipative semiclassical systems
}

\author{
Bidhan Chandra Bag and Deb Shankar Ray* \\ Indian Association for the Cultivation of Science, Jadavpur, Calcutta 700 032, INDIA.
}

\begin{abstract}
We examine the weak noise limit of an overdamped dissipative system within a semiclassical description and show how quantization influences the growth and decay of fluctuations of the thermally equilibrated systems. We trace its origin in a semiclassical counterpart of the generalized potential for the dissipative system.
\end{abstract}

Keywords : Dissipative quantum systems, large fluctuations, generalized potential.

PACS number(s): 05.40.-a, 05.70.Ln

*pcdsr@mahendra.iacs.res.in 


\section{INTRODUCTION}

One of the important issues in nonequilibrium phenomena in the macroscopic nonlinear systems is to understand the interplay of nonlinearity of the system and the fluctuations of its environment. The problem is fairly general in the context of chemical reactions [1], breakdown of electronic devices [2], phase transitions [3] etc. The essential description of the physical situation rests on the Fokker-Planck equations for the probability distribution functions of the relevant variables of the dynamics. In the weak noise limit the fluctuations have been described [3 [6] by appropriate auxiliary Hamiltonian or path integral methods. The theoretical results have been corroborated by remarkable experiments on fluctuations using analogue electronic circuits [3], which allow the phase space trajectories of fluctuations to be observed directly in a precise manner. These studies have enriched our understanding in several theoretical issues, e. g. , the symmetry between the growth and the decay of classical fluctuations in equilibrium and its breakdown under nonequilibrium conditions [3] , the existence of a nonequilibrium potential of a dissipative system [5.6] etc.

It is the purpose of this paper to extend the theory to the semiclassical context. The quantization of the system itself adds a new dimension to the interplay of nonlinearity and stochasticity in a dissipative system. To make a fair comparison with classical theory we adopt the Wigner's phase space distribution function of c-number variables. The weak noise limit can then be appropriately employed to develop an auxiliary Hamiltonian formulation at the semiclassical level in terms of these phase space variables. This allows us to realize the existence of an optimal force of purely quantum origin derivable from the fluctuating field and relate it to the momentum of the auxiliary Hamiltonian. The quantum correction also makes its presence felt in the growth and decay of fluctuations of thermally equilibrated semiclassical systems keeping the symmetry preserved.

The outline of the paper is as follows: In Sec. II we introduce the general aspects of dynamics of dissipative quantum system in terms of the Wigner phase space function. In Sec. III we consider the weak noise and semiclassical limit under overdamped condition and 
take resort to the well-known auxiliary Hamiltonian description. The quantum part of the optimal force is then explicitly derived. The symmetry between the growth and decay of fluctuations in a thermalized quantum system is discussed in Sec. IV. The existence of a semiclassical contribution to the potential in a dissipative system is then shown in Sec. V. The paper is concluded in Sec. VI.

\section{QUANTUM DYNAMICS OF A DISSIPATIVE SYSTEM}

We consider a dynamical system characterized by a potential $V(x)$ coupled to an environment. Evolution of such an open quantum system has been studied over the last several decades under a variety of reasonable assumptions [0] 9]. Specifically interesting is the semiclassical limit of an Ohmic environment. The dissipative time evolution of the Wigner distribution function $W(x, p, t)$ for the system with unit mass $(m=1)$ under the potential $V(x)$ can be described by 10,12

$$
\frac{\partial W}{\partial t}=-p \frac{\partial W}{\partial x}+\frac{\partial V}{\partial x} \frac{\partial W}{\partial p}+\epsilon \sum_{n \geq 1} \frac{\hbar^{2 n}(-1)^{n}}{2^{2 n}(2 n+1) !} \frac{\partial^{2 n+1} V}{\partial x^{2 n+1}} \frac{\partial^{2 n+1} W}{\partial p^{2 n+1}}+\gamma \frac{\partial p W}{\partial p}+D \frac{\partial^{2} W}{\partial p^{2}}
$$

where $\gamma$ and $D$ are the dissipation constant and the diffusion coefficient, respectively. $x$ and $p$ are c-number co-ordinate and momentum variables. The drift term is a direct consequence [8] of the existence of $\gamma$-dependent term in the imaginary part of the exponent in the expression for the propagator for the density operator in the Feynman-Veron theory and has been shown to be responsible for appearance of a damping force in the classical equation of motion for the Brownian particle to ensure quantum-classical correspondence. $\gamma$ and D are related by the fluctuation-dissipation relation, $D=\frac{\gamma}{2} \hbar \omega \operatorname{coth} \frac{\hbar \omega}{2 k_{b} T}$ (in the semiclassical limit $D=\gamma k T$

). $\omega$ is the renormalised linear frequency of the nonlinear system. The quantum correction to classical Liouville motion is contained in the $\hbar$-containing terms in the sum. $\epsilon$ is a parameter (whose value is 1) which is kept in the equation for bookkeeping the Wigner correction term in our further analysis. We put $\epsilon=1$ at the end of calculation.

Eq.(1) had been used earlier in several occasions. For example, Zurek and Paz [10] and others [12] have studied some interesting aspects of quantum-classical correspondence in 
relation to decoherence and chaos. Based on this equation and its variant chaotic dissipative systems has been studied. [11,13, 15]. The equation also yields the simplest leading order quantum correction term to classical Kramers' rate [16]. The primary reason for choosing Eq.(3) as our starting point is that it reaches the correct classical limit when $\hbar \rightarrow 0$ so that $D$ becomes a thermal diffusion coefficient $(\gamma k T)$ in the high temperature limit and the Wigner function reduces to the corresponding classical phase space distribution function and we recover the Kramers' equation which describes classical Brownian motion of a particle in phase space.

\section{WEAK NOISE AND SEMICLASSICAL LIMIT OF QUANTUM DISSIPATIVE DYNAMICS:}

The weak noise limit of a dissipative system within a semiclassical description can be conveniently described by a "WKB-like" ansatz (we refer to "WKB-like" since we are considering more than one dimension. Traditionally WKB refer to one dimension only) of the Eq.(1) for the Wigner function of the form

$$
W(x, p, t)=Z(x, t) \exp \left(-\frac{x p}{\hbar}\right) \exp \left(-\frac{s}{D_{1}}\right)
$$

where $D_{1}=\frac{D}{\omega^{2}}$. The weak noise limit is defined [3] as $D_{1} \rightarrow 0$ and semiclassical limit refers to $(\hbar \rightarrow 0)$. $Z(x, t)$ is a prefactor and $s(x, p, t)$ is a classical action which is a function of c-number variables $x$ and $p$, satisfying the following Hamilton-Jacobi equation,

$$
\begin{aligned}
& \frac{\partial s}{\partial t}+p \frac{\partial s}{\partial x}-V^{\prime} \frac{\partial s}{\partial p}-\gamma p \frac{\partial s}{\partial p}+\omega^{2}\left(\frac{\partial s}{\partial p}\right)^{2} \\
& +\epsilon \sum_{n \geq 1} \frac{x^{2 n}(-1)^{3 n+1}}{2^{2 n}(2 n) !} \frac{\partial^{2 n+1} V}{\partial x^{2 n+1}} \frac{\partial s}{\partial p}=0 .
\end{aligned}
$$

The derivation of Eq.(3) is based on the following consideration. Since in the weak noise limit $D_{1}$ is the relevant small parameter one obtains with ansatz (2) in leading order a term proportional to $\hbar^{2 n}\left(\frac{1}{D_{1}} \frac{\partial s}{\partial p}\right)^{2 n+1}$ which is not balanced by any other term of the same order

$D_{1}{ }^{-(2 n+1)}$. This is because the highest derivative in Eq.(1) does not have a factor scaling 
with the corresponding power of $D_{1}$. The successive terms next to the leading order are also $\hbar$-containing terms. All of these terms vanishes in the semiclassical limit $(\hbar \rightarrow 0)$. Therefore the leading order term that remains gives rise to Eq.(3). It is thus obvious that an ansatz (2) with $\hbar$ finite is not feasible. The semiclassical limit $\hbar \rightarrow 0$ is a necessary requirement for the validity of ansatz (2). The above equation can be solved by integrating the Hamiltonian equations of motion,

$$
\begin{aligned}
\dot{x} & =p \\
\dot{X} & =P-\gamma X \\
\dot{p} & =V^{\prime}+\gamma p-2 \omega^{2} X-\epsilon \sum_{n \geq 1} \frac{(-1)^{3 n+1} x^{2 n}}{2^{2 n}(2 n) !} \frac{\partial^{2 n+1} V}{\partial x^{2 n+1}} \\
\dot{P} & =\left[V^{\prime \prime}-\epsilon \sum_{n \geq 1} \frac{(-1)^{3 n+1}}{2^{2 n}(2 n) !} \frac{\partial}{\partial x}\left(x^{2 n} \frac{\partial^{2 n+1} V}{\partial x^{2 n+1}}\right)\right] X
\end{aligned}
$$

which are derived from the following effective Hamiltonian $H_{\text {eff }}$

$$
\begin{aligned}
H_{e f f}= & p P-V^{\prime} X-\gamma X p+\omega^{2} X^{2} \\
& +\epsilon \sum_{n \geq 1} \frac{x^{2 n}(-1)^{3 n+1}}{2^{2 n}(2 n) !} \frac{\partial^{2 n+1} V}{\partial x^{2 n+1}} X .
\end{aligned}
$$

Here we have put $\frac{\partial s}{\partial x}=P$ and $\frac{\partial s}{\partial p}=X$. The introduction of additional degree-of-freedom by incorporating the auxiliary momentum $(\mathrm{P})$ and co-ordinate $(\mathrm{X})$ makes the system an effectively two-degree-of-freedom system. The origin of these two variables is the thermal fluctuations of the environment [3]. The auxiliary Hamiltonian $H_{\text {eff }}$ is not to be confused with the microscopic Hamiltonian comprising the system, the bath and their coupling. Thus the phase space trajectories concern fluctuations of the c-number variables.

Under overdamped condition $(\ddot{x} \ll \gamma \dot{x} ; \ddot{X} \ll \gamma \dot{X})$ Eqs. (4) can be easily reduced to the following form ;

$$
\begin{aligned}
& \dot{x}=K(x)+\frac{2 \omega^{2} X}{\gamma} \\
& \dot{X}=-\frac{\partial K(x)}{\partial x} X
\end{aligned}
$$

where 


$$
K(x)=\frac{1}{\gamma}\left[-V^{\prime}+\epsilon \sum_{n \geq 1} \frac{(-1)^{3 n+1} x^{2 n}}{2^{2 n}(2 n) !} \frac{\partial^{2 n+1} V}{\partial x^{2 n+1}}\right]
$$

It is easy to recognize the quantity $\frac{2 \omega^{2} X}{\gamma}$ as a momentum and redefine it as $p_{r}$. Therefore $\operatorname{Eqs}(6)$ may be rewritten as

$$
\begin{aligned}
\dot{x} & =K(x)+p_{r} \\
\dot{p_{r}} & =\frac{\partial K(x)}{\partial x} p_{r} .
\end{aligned}
$$

So the overdamped motion is described by the following effective Hamiltonian,

$$
H_{o d}=\frac{p_{r}^{2}}{2}+K(x) p_{r}
$$

The above auxiliary Hamiltonian description $(8,9)$ is isomorphic in form to that of Luchinsky and McClintock [3], who had considered an overdamped classical Brownian motion in a force field $K(x)$, driven by a weak white noise $\zeta(t)$ whose intensity $D_{1} \ll 1$ as

$$
\dot{x}=K(x)+\zeta(t),\langle\zeta\rangle=0,\langle\zeta(t) \zeta(0)\rangle=D_{1} \delta(t)
$$

Equivalently the corresponding Fokker-Planck equation for the probability density $P(x, t)$ is

$$
\frac{\partial P(x, t)}{\partial t}=-\frac{\partial K(x)}{\partial x} P(x, t)+\frac{D_{1}}{2} \frac{\partial^{2} P(x, t)}{\partial x^{2}} .
$$

The large fluctuations of scale $\gg \sqrt{D_{1}}$ can therefore be treated in the weak noise limit $D_{1} \rightarrow 0$ [3, 5],6] by "WKB like" approximation of the Fokker-Planck equation (11) in the form

$$
P(x, t)=y(x, t) \exp \left[\frac{-\phi(x, t)}{D_{1}}\right]
$$

Here $\mathrm{y}(\mathrm{x}, \mathrm{t})$ is the prefactor and $\phi(x, t)$ is a "classical" action describing a HamiltonianJacobi equation which can be solved by solving Hamilton' s equation (8) with $p_{r}=\frac{\partial \phi}{\partial x}$ as the momentum for auxiliary system. 
The important distinguishing feature of the above description in which the system is treated semiclassically is the structure of $K(x)$ which is given by equation( 7$)$ and comprises of two terms ;

$$
K(x)=K_{c l}+K_{s e m i}(x)
$$

where

$$
K_{c l}=-\frac{V^{\prime}(x)}{\gamma}
$$

is derivable from purely classical potential $V(x)$ and $K_{s e m i}(x)$ does not explicitly involve $\hbar$

$$
K_{s e m i}(x)=\frac{\epsilon}{\gamma} \sum_{n \geq 1} \frac{x^{2 n}(-1)^{3 n+1}}{2^{2 n}(2 n) !} \frac{\partial^{2 n+1} V}{\partial x^{2 n+1}}
$$

originates from the nonlinearity of the potential $V(x)$ and quantum nature of the system. The quantum contribution to $K(x)$ is therefore likely to influence both the fluctuational and the relaxational paths of the dynamics and is also responsible for the existence of a potential. Our objective is now to explore these aspects in the following two sections.

\section{LARGE FLUCTUATIONS IN EQUILIBRATED SEMICLASSICAL SYSTEMS}

In the thermally equilibrated systems a typical large fluctuation of the variable $x$ implies a temporary departure from its stable state, $x_{s}$ to some remote state $x_{f}$. This is followed by a return to $x_{s}$ as a result of relaxation in the absence of fluctuations $p_{r}$. A nonzero value of $p_{r}$ which results from fluctuations due to surrounding drives the system away from $x_{s}$ along a set of trajectories which form the unstable invariant manifold and define the so called fluctuational paths. On the other hand the system relaxes along the relaxational return path to $x_{s}$ under the condition $p_{r}=0$, which form stable invariant manifold. The latter condition implies $\dot{x}=K(x)$. In each case the trajectory represents the optimal paths

along which the system is expected to move with overwhelming probability. Luchinsky and McClintock [3] have studied these paths in analog electronic circuits and demonstrated the 
growth and the decay of classical fluctuations in equilibrium. We extend this analysis to semiclassical domain using the same model potential,

$$
V(x)=\frac{1}{4} x^{4}-\frac{1}{2} x^{2}
$$

The quantum contribution to the growth and the decay of fluctuations can be understood by recognizing the $K_{\text {semi }}(x)$ term in the dynamics (8). In Fig.1 we compare both the deterministic fluctuational and relaxational (optimal) paths for quantum and classical thermally equilibrated systems. It is important to note that the maximum possible amplitude of large fluctuations is almost double for the quantum system compared to that for the corresponding the classical system. This is due to the addition of the nonlinear force term

of quantum origin, $K_{\text {semi }}(x)$ in the c-number equation (8), which is shown to be derivable from the fluctuating field, and is related to the momentum of the auxiliary Hamiltonian.

Before leaving this section we make a brief remark on the thermally nonequilibrated systems. Since the detailed balance is not operative here, the optimal path to a given state not just the time-reversed dynamical path along which the system moves from this state to the stable state in absence of fluctuations $p_{r}$. Thus for the driven system, for example, the pattern of optimal path is generically different from that for the thermally equilibrated systems. It may display singularities whose topological manifestations as caustics, switching line, cusps etc have been thoroughly studied for classical systems. We believe that $K_{\text {semi }}(x, t)$ where $\mathrm{t}$ signifies the driving by a periodic force in $V(x, t)$ is likely to play an important role in their quantum counterparts.

\section{EXISTENCE OF A POTENTIAL FOR DISSIPATIVE SEMICLASSICAL SYSTEM}

In a significant analysis Graham and coworkers [5, 6] had examined the condition for existence of potential for classical dissipative systems. We now extend this analysis to the present semiclassical context. 
The general criterion for a dissipative dynamical system described by autonomous equations of the form

$$
\dot{x}^{\nu}=K^{\nu}(x)
$$

to have a potential $\phi(x)$ with respect to $Q^{\nu \mu}$ (positive, semidefinite symmetric matrix, which are considered to be the matrix of transport coefficients) if there exists a single-valued continuously differentiable and globally defined function $\phi(x)$, bounded from below which is stationary in the limit sets of Eq.(17) and which satisfies

$$
K^{\nu}(x)=-\frac{1}{2} Q^{\nu \mu} \frac{\partial \phi(x)}{\partial x^{\mu}}+r^{\nu}(x)
$$

with

$$
r^{\nu} \frac{\partial \phi(x)}{\partial x^{\nu}}=0
$$

Here for simplicity $Q^{\nu \mu}$ is assumed to be independent of $x$. The first and the second terms of Eq.(18) correspond to irreversible and reversible part, respectively.

The stochastic process $x\left(D_{1}, t\right)$ which involve Eq.(17) and a symmetric non-negative matrix $Q^{\nu \mu}$ is governed by the Fokker-Planck equation for probability distribution function $P(x, t)$

$$
\frac{\partial P(x, t)}{\partial t}=-\frac{\partial}{\partial x^{\nu}} K^{\nu}(x) P+\frac{D_{1}}{2} \frac{\partial^{2}}{\partial x^{\nu} \partial x^{\mu}} Q^{\nu \mu} p
$$

For $D_{1}=0$ the above description reduces to $(17)$. For $D_{1} \neq 0$ the steady state distribution defines the function $\phi(x, t)$ by

$$
P\left(x, D_{1}, t \rightarrow \infty\right)=N\left(D_{1}\right) \exp \left[-\frac{\phi\left(x, D_{1}\right)}{D_{1}}\right]
$$

$\mathrm{N}$ is the normalization constant. If $\phi(x)=\lim _{D_{1} \rightarrow 0} \phi\left(x, D_{1}\right)$ is a single-valued, continuously differentiable function bounded from below it satisfies

$$
K^{\nu}(x) \frac{\partial \phi(x)}{\partial x^{\nu}}+\frac{1}{2} Q^{\nu \mu} \frac{\partial \phi(x)}{\partial x^{\nu}} \frac{\partial \phi(x)}{\partial x^{\mu}}=0
$$


Eq. (22) is equivalent to Eqs. (18, 19). Interpreting Eq.(22) as usual as a HamiltonJacobi equation by defining $\phi(x)$ as action and $\frac{\partial \phi}{\partial x^{\nu}}=P_{\nu}$ as the momentum conjugate to $x_{\nu}$, one can construct the auxiliary Hamiltonian

$$
H(x, p)=\frac{1}{2} Q^{\nu \mu} p_{\nu} p_{\mu}+K^{\nu}(x) p_{\nu}
$$

Graham and co-workers [5,6] have argued that a potential can exist with equation (17) if the above Hamiltonian is integrable for $H=0$, because the condition implies that there exist a smooth separatrix which connect smoothly the stable and unstable manifolds emanating from the hyperbolic fixed points of the dynamical system.

We now turn back to our dissipative semiclassical system described by Eqs(8) and (9) where $K(x)$ is defined by Eq.(7). Recognizing Eq.(9) as Eq.(23) for a one-degree-of-freedom system we identify

$$
\begin{aligned}
Q & =1 \\
K(x) & =K_{c l}+K_{s e m i} .
\end{aligned}
$$

The potential function $\phi(x)$ can therefore be calculated from Eq.(9) with $H=0$ as (since $\left.p_{r}=\frac{\partial \phi(x)}{\partial x}\right)$

$$
\phi(x)=-2 \int K(x) d x
$$

The above expression can be made more explicit if we make use of Eq.(7) in (25). We obtain

$$
\phi(x)=\phi_{c l}(x)+\phi_{\text {semi }}(x)
$$

where

$$
\phi_{c l}=\frac{2}{\gamma} \int V^{\prime}(x) d x=\frac{2}{\gamma} V(x)
$$

and

$$
\phi_{\text {semi }}(x)=-\frac{2}{\gamma} \sum_{n \geq 1} \int \frac{x^{2 n}(-1)^{3 n+1}}{2^{2 n}(2 n) !} \frac{\partial^{2 n+1} V}{\partial x^{2 n+1}} d x
$$


The existence of a potential for a dissipative, semiclassical dynamical system is thus ascertained. The method essentially relies on a dynamical definition $p_{r}$ as a derivative of the potential $\phi(x)$ in a system described by an overdamped quantum Markov process in the weak noise and semiclassical limit. As elaborated earlier in Sec.III $p_{r}$ has a statistical origin which drives the system away from its stable state $x_{s}$ to a preassigned remote state $x_{f}$ from which the system relaxes in absence of $p_{r}$. It is thus important to realize that both the dynamical and statistical notions are kept intact in the quantum treatment.

\section{CONCLUSIONS}

Keeping in view of the quantum nature of the system in terms of the Wigner's phase space function we examine the semiclassical dynamics of a dissipative system in an Ohmic environment. The weak noise limit of the stochastic process then allows us to capture the essential features of the dynamics within the framework of an auxiliary Hamiltonian description at the semiclassical level. Our results are summarized as follows :

(i) The Wigner's quantum correction to classical Liouville equation gives rise to an optimal force in addition to usual the classical force term. This quantum optimal force is essentially a result of an interplay of nonlinearity of the system and the thermal fluctuations of its environment and is derivable in terms of an auxiliary Hamiltonian description.

(ii) This term is also responsible for modification of growth and decay of large fluctuations from equilibrium for the appropriately thermalized quantum system (compared to its classical counterpart). The symmetry of the fluctuational and the relaxational paths, signifying the detailed balance, however, as expected is kept preserved.

(iii) The quantum correction term implies the existence of a potential for the dissipative semiclassical system. 
Since the fluctuational and the relaxational paths have been experimentally demonstrated as a part of physical reality by analogue experiments [3] in the realm of large fluctuations, we believe that the essential modification of the integrable, nonintegrable and the singular

topological features of the dynamics due to semiclassical correction might be relevant in several contexts. We hope to address some of these issues in a future communication.

\section{ACKNOWLEDGMENTS}

B. C. Bag is indebted to the Council of Scientific and Industrial Research for a fellowship. 


\section{REFERENCES}

[1] M. I. Dykman, E. Mori, J. Ross and M. P. Hunt, J. Chem. Phys. 100, 5737 (1994).

[2] R. L. Kautz, Rep. Prog. Phys. 59, 935 (1996).

[3] D. G. Luchinsky and P. V. E. McClintock, Nature 389, 403 (1997).

[4] A. J. Bray and A. J. McKane, Phys. Rev. Lett. 62, 493 (1989).

[5] R. Graham, in Noise in Nonlinear Dynamical Systems Vol. 1 (eds F. Moss and P. V. E. McClintock) 225-278 (Cambridge Univ. Press, 1989).

[6] R. Graham and T. Tél, Phys. Rev. Lett 52, 9 (1984).

[7] W. H. Louisell, Quantum Statistical Properties of Radiation (Wiley, New York, 1973); G. W. Ford, J. T. Lewis and R. J. O’Connell, Ann. Phys.(N.Y.) 185, 270 (1988).

[8] A. J. Leggett and A. O. Caldeira, Physica A121, 587 (1983).

[9] See, for example, the review by G. Gangopadhay and D. S. Ray, in Advances in Multiphoton Processes and Spectroscopy Vol 8, edited by S. H. Lin, A. A. Villayes and F. Fujimura, (World Scientific, Singapore, 1993).

[10] W. H. Zurek and J. P. Paz, Phys. Rev. Lett. 72, 2508 (1994) ; S. Habib, K. Shizume and W. H. Zurek, Phys. Rev. Lett. 80, 4361 (1998).

[11] B. C. Bag, S. Chaudhuri, J. Ray Chaudhuri and D. S. Ray, Physica D 125, 47 (1999).

[12] A. K. Pattanayak, Phys. Rev. Lett. 83, 4526 (1999).

[13] M. E. Goggin, B. Sundaram and P. W. Milonni, Phys. Rev. A 41, 5705 (1990).

[14] T. Dittrich and R. Graham, Ann. Phys. 200, 363 (1990)

[15] B. C. Bag and D. S. Ray, J. Stat. Phys. 96, 271 (1999).

[16] J. Ray Chaudhuri, B. C. Bag and D. S. Ray, J. Chem. Phys. 111, 10852 (1999). 


\section{FIGURES}

FIG. 1. A plot of system co-ordinate $x$ vs time $t$ signifying the fluctuational and relaxational

paths according to Eq.(8) for the model system with $V(x)=\frac{1}{4} x^{4}-\frac{1}{2} x^{2}$ for $(a)$ semiclassical case $(\epsilon=1)$ and (b) classical case $(\epsilon=0)$, (units arbitrary). 
B. C. Bag and D. S. Ray

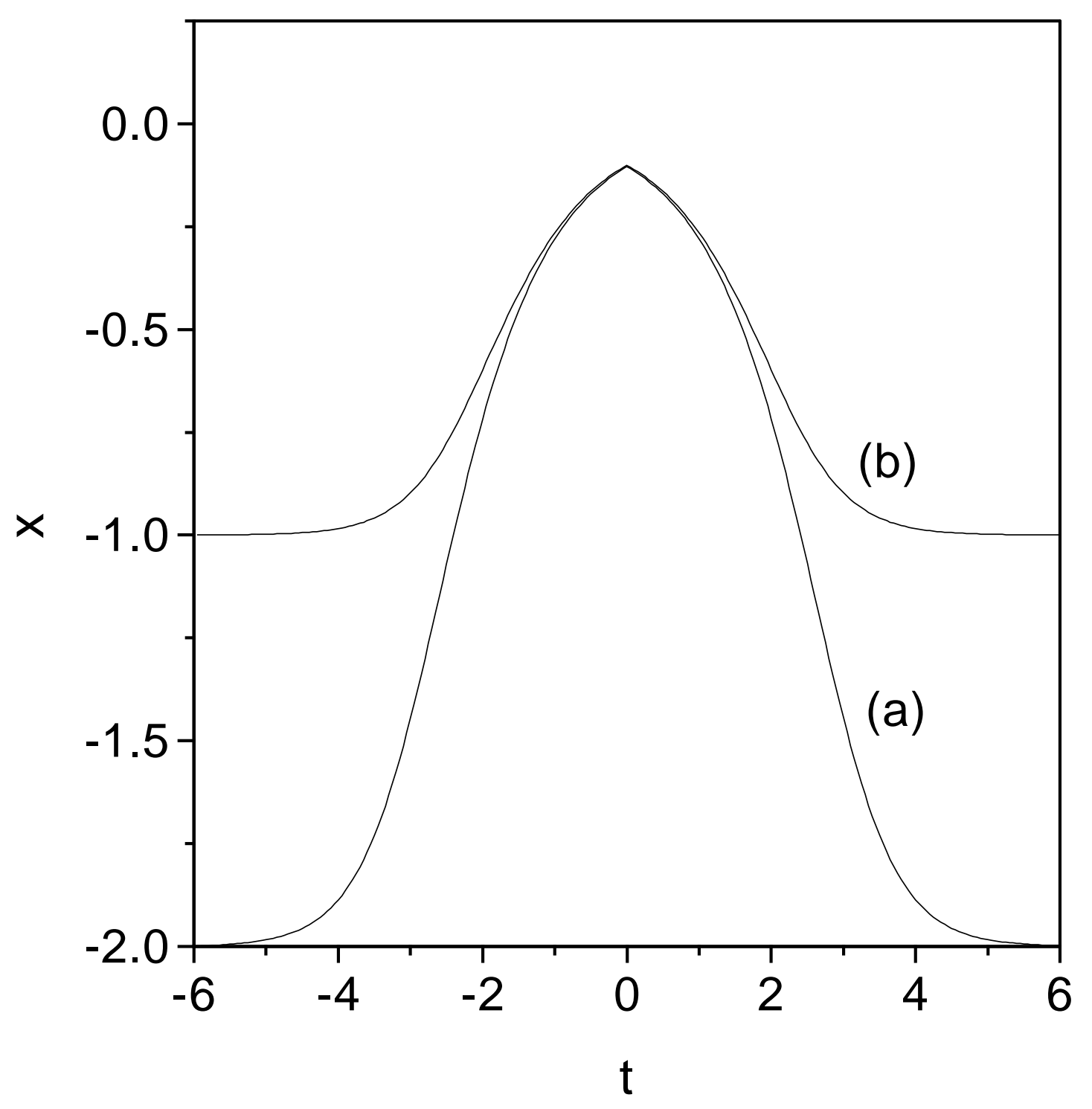

Fig. 1 